\title{
Cuidados de enfermagem ao idoso com Demência Que apresenta sintomas comportamentais
}

\author{
Nursing care for the aged with Dementia and with behavioral symptoms \\ La atención de enfermería a los ancianos con Demencia y con síntomas comportamentales
}

\section{Luana Cardoso Pestana', Célia Pereira Caldas'}

'Universidade do Estado do Rio de Janeiro. Faculdade de Enfermagem. Rio de Janeiro, RI

Submissão: $03 / 09 / 2008$

Aprovação: 16/06/2009

\section{RESUMO}

No curso das demências, os transtornos comportamentais são altamente prevalentes e estão freqüentemente associados ao stress dos cuidadores. Este estudo tem como objetivo identificar na literatura os tipos de demências que apresentam sintomas comportamentais e psicológicos associados; descrever o manejo não farmacológico destes distúrbios e descrever a atuação da enfermagem neste contexto. Foi pesquisada a literatura nas áreas de geriatria e enfermagem. Tornou-se evidente que a assistência de enfermagem prestada ao idoso demenciado vai muito além dos cuidados básicos e depende da participação ativa da família. A identificação de estratégias para minimizar e manejar os sintomas comportamentais é uma importante contribuição Que a enfermagem pode dar para a geriatria.

Descritores: Demência; idoso; Cuidadores; Enfermagem; Geriatria.

\begin{abstract}
In the course of dementia, behavioral disorders are highly prevalent and are frequently associated with the stress of caregivers. This study aims to identify in scientific literature the types of dementia associated with behavioral and psychological symptoms (BPSD); describe the non-pharmacological management of these alterations, and describe the nursing interventions in this context. The literature in the field of geriatrics and nursing was searched. It became evident that the nursing care provided to the demented elderly goes farther beyond the basic care and depends on the active participation of the family. The identification of strategies to minimize and manage the behavioral symptoms is an important contribution that nursing can give to geriatrics.
\end{abstract}

Descriptors: Dementia; Aged; Caregivers; Nursing; Geriatrics.

\section{RESUMEN}

En el curso de la demencia, los trastornos de comportamiento son muy prevalentes y se asocian frecuentemente con el estrés de los cuidadores. Este estudio tiene como objetivo identificar en la literatura científica los tipos de demencias asociadas a los trastornos de comportamiento y síntomas psicológicos, describir el manejo no-farmacológico de estas alteraciones y describir las intervenciones de enfermería en este contexto. La Literatura en los campos de la geriatría y de enfermería fue investigada. Se hizo evidente Que los cuidados de enfermería prestados a las personas mayores con demencia va más allá de la atención básica y depende de la participación activa de la familia. La identificación de estrategias para minimizar y manejar los síntomas de comportamiento es una importante contribución Que la enfermería puede ofrecer a la geriatría.

Descriptores: Demencia; Cuidadores; Enfermería; Geriatría. 


\section{INTRODUÇÃO}

Este artigo tem como eixo principal a assistência de enfermagem ao idoso demenciado e seus cuidadores. A análise se alicerça em conteúdo teórico-conceitual, através de revisão de literatura extensiva, na área de geriatria, gerontologia e enfermagem. A escolha deste tema se deu porque a demência tem se revestido de uma maior importância como problema de saúde devido ao aumento do contingente da população envelhecida em todo mundo, particularmente a faixa etária dos $80 \operatorname{anos}^{(1)}$. Outros estudos sinalizam Que os distúrbios demenciais são a principal causa de incapacidade na velhice. E acrescentam Que, há fortes indícios Que a demência atualmente se situe entre as doenças que mais matam ${ }^{(2)}$.

A demência é hoje considerada uma síndrome clínica caracterizada por declínio cognitivo, com caráter permanente e progressivo ou transitório, causada por múltiplas etiologias, acarretando repercussões sociais e ocupacionais ao paciente ${ }^{(3)}$. Dentre as alterações cognitivas incluem-se distúrbios de memória, de linguagem, alterações nas capacidades visoespaciais, de autocuidado, de fazer julgamentos e de pensamento abstrato.

No curso das demências, transtornos não-cognitivos comportamentais são altamente prevalentes ${ }^{(4)}$. Estes transtornos receberam, em 1996, a denominação de sintomas psicológicos e comportamentais da demência (SPCD), por Finkel et al(5) após consenso do International Psychogeriatric Association (IPA). A terminologia refere-se ao conjunto de sintomas e sinais relacionados a transtornos de percepção, do conteúdo do pensamento, do humor ou do comportamento Que ocorrem freqüentemente em pacientes com síndrome demencial.

Sendo a demência reconhecidamente um processo irreversível de deterioração progressiva em Que a intervenção farmacológica tal Qual a institucionalização não garantem efetivos benefícios, a assistência prestada pelo cuidador familiar ou formal mostra-se em diversos estudos associada ao desgaste físico e emocional de Quem cuida, evidenciando uma sobrecarga ainda maior diante do idoso demenciado Que apresenta sintomas não- $\operatorname{cognitivos}^{(6)}$.

Em 2007, participando de treinamento profissional em gerontogeriatria passei a realizar consultas de enfermagem junto aos idosos e seus cuidadores. Diante do diagnóstico de demência observei Que dentro das atividades exercidas por mim na assistência, o Que garantiria melhor Qualidade de vida aquele idoso eram as minhas orientações sobre o cuidado, uma vez Que, a sobrecarga física e emocional do cuidado a um familiar demenciado é imensa e requer a execução de técnicas específicas de enfermagem como mudança de decúbito, banho, alimentação, cuidados com a pele, etc. Não obstante, as demandas familiares, por diversas vezes, eram em torno do manejo do idoso demenciado com transtornos comportamentais.

Desta forma, a sobrecarga física, emocional e sócio-econômica do cuidado de um familiar é imensa. E não se pode exigir Que os cuidados sejam corretamente executados sem Que haja orientação desses responsáveis. É fundamental Que profissionais de saúde treinem o cuidador e supervisione a execução das atividades assistenciais necessárias ao cotidiano do idoso até que a família se sinta segura para assumi-la ${ }^{(7)}$.

Assim, emergiu a necessidade de buscar na literatura científica conhecimentos sobre as demências, os SPCD, manejo nãofarmacológico desses sintomas e como a enfermagem gerontológica tem atuado diante dessa clientela.

Portanto, o objeto deste estudo é a assistência de enfermagem ao idoso demenciado Que apresenta sintomas comportamentais e psicológicos da demência, tendo como Questões norteadoras: Quais são os sintomas comportamentais e psicológicos Que idosos demenciados geralmente apresentam? Qual é a atuação do enfermeiro junto ao idoso e seu cuidador neste contexto?

Para a realização do estudo foi estruturado como objetivo geral realizar revisão de literatura extensiva na área de geriatria, gerontologia e enfermagem, acerca da assistência de enfermagem ao idoso demenciado Que vivencia sintomas comportamentais e psicológicos. E, como objetivos específicos: identificar na literatura os tipos de demências senis Que apresentam, geralmente, sintomas comportamentais e psicológicos associados; descrever o que a literatura, nos últimos sete anos, apresenta como consenso no manejo não farmacológico dos SCPD; descrever a atuação da enfermagem gerontológica junto ao idoso e seu cuidador neste contexto.

Através deste estudo, espera-se contribuir na atualização de conhecimentos para as áreas de enfermagem gerontológica, levantando a temática para reflexão dos profissionais Que lidam com esta clientela na busca, tão necessária, da construção de um modelo de atenção adeQuado.

\section{METODOLOGIA}

O estudo foi desenvolvido a partir de revisão de literatura realizada através da Biblioteca Virtual em Saúde - Sistema Bireme - com buscas nos seguintes diretórios: LILACS (Literatura LatinoAmericana e do Caribe em Ciências da Saúde), SCIELO (Scientific Eletronic Library Online), BDENF (Base de Dados de Enfermagem) e MEDLINE (Literatura Internacional de Ciências em Saúde).

Utilizaram-se as palavra: demência, transtornos de comportamento, tratamento, cuidadores, família, enfermagem.

\section{RESULTADOS E DISCUSSÃO}

\section{Sintomas Comportamentais e Psicológicos das Demências}

O processo de envelhecimento é, em sua essência, multifatorial. Ele depende da programação genética e das alterações Que ocorrem em nível celular e molecular Que resultam em sua aceleração ou desaceleração, redução de massa celular ativa, diminuição da capacidade funcional e sobrecarga dos mecanismos de controle homeostático.

O envelhecimento cerebral fisiológico ocorre através de mudanças morfofuncionais, bioquímicas e nos próprios neurotransmissores Quando ocorre a perda ponderal do cérebro, diminuição do número de neurônios em certas áreas, ateromatose de vasos cerebrais, formação de placas neuríticas e a diminuição de neurotrasmissores/ enzimas como a acetilcolina, serotonina e catecolaminas.

São tantas as perdas, Que fica difícil diferir o "normal" do Que é patológico no processo de envelhecer. Não existem critérios rígidos para estabelecer o limite entre os estados fisiológico e patológico o Que pode vir a retardar o diagnóstico ou incorrer em erros. Como essas mudanças podem ocorrer de forma concomitante é necessário avaliar o idoso de forma global e buscar precocemente diagnosticar as situações potencialmente reversíveis, através de uma anamnese 
bem detalhada, das informações fornecidas por familiares e/ou cuidadores e de métodos complementares de diagnóstico ${ }^{(8)}$.

Entre todos os processos mórbidos que acometem o idoso, principalmente em fases mais avançadas da existência, a demência, em suas diversas formas, tem particular relevância, não só pela freqüência com Que ocorrem, mas principalmente, por ser, provavelmente, a mais devastadora das entidades patológicas, com sua ação deletéria sobre o idoso, a família, cuidadores e na sociedade em geral ${ }^{(3)}$.

As síndromes demenciais são caracterizadas pela presença de déficit cognitivo progressivo, com maior ênfase na perda de memória e interferência nas atividades sociais e ocupacionais. Segundo pesquisa feita por Jorm, em 1987, a incidência e a prevalência das demências aumenta exponencialmente com a idade, dobrando, aproximadamente, a cada cinco anos a partir dos 60 anos de idade. Assim, a prevalência das demências na população de idosos entre 65-69 anos de idade é de 1,4\%; de 85-89 anos de idade, 20,8\%; e dos $90-95$ anos, de $38,6 \%{ }^{(9)}$.

Dentro do diagnóstico das síndromes demenciais a Doença de Alzheimer (DA) aparece como primeira causa de demência em diversos estudos, correspondendo de 50-70\% dos $\operatorname{casos}^{(3-6,8-1)}$. A demência vascular (DV) é a segunda forma de demência mais comum, configurando-se em aproximadamente $20 \%$ dos casos. A Demência vascular pode estar presente na Doença de Alzheimer denominada Demência Mista, mas não encontrei dados sobre prevalência e incidência referentes a esta forma de demência. Outras formas de demências como a Demência por Corpúsculos de Lewy (DCL) também são citadas como 15-20\% dos casos diagnosticados ${ }^{(3,10)}$.

De fato, a deterioração das funções cognitivas constitui o núcleo fundamental dos sintomas clínicos das demências. Mas, existem muitos outros sintomas Que podem afetar gravemente a Qualidade de vida dos pacientes e gerar numerosos problemas, a eles e aos seus cuidadores. Estes sintomas, Que podem ser classificados como secundários, são muito diversos - são os sintomas comportamentais e psicológicos das demências (SCPD). Estudos sugerem Que os sintomas mais freqüentes são os sintomas psicóticos (delírios e alucinações), agitação, depressão e distúrbios do sono ${ }^{(11,12)}$. Uma revisão abrangendo 55 estudos entre 1990-2003 concluiu Que na Doença de Alzheimer os sintomas psicóticos correspondem a maior síndrome clínica da doença, ocorrendo em 41\% dos indivíduos após três anos do diagnóstico de DA. Destes, 36\% apresentaram delírios, 18\% alucinações e $25 \%$ os demais sintomas ${ }^{(12)}$. Estes números variam bastante, dependendo da amostra estudada e dos métodos de avaliação. Estudos estrangeiros voltados para os SCPD em pacientes com diagnóstico de DA revelaram a presença de sintomas neuropsiquiátricos em 75-89\% de suas amostras ${ }^{(4,15)}$, corroborando com o estudo paulista Que evidenciou em 78,3\% dos pacientes com DA, sintomas neuropsiquiátricos ${ }^{(16)}$.

A presença dos sintomas comportamentais e psicológicos em idosos demenciados afeta não só os pacientes, mas a família e cuidadores formais ou informais. Sua ocorrência tem sido relacionada ao curso clínico desfavorável, evolução rápida da doença, aumento da taxa de institucionalização, aumento da sobrecarga dos cuidadores e diminuição da sobrevida desses pacientes ${ }^{(4,12,17-19)}$.

\section{Tratamento dos SCPD}

A partir da década de 90 , houve o aumento do número de estudos avaliando o impacto de abordagens terapêuticas farmacológicas e não-farmacológicas nos SCPD. Apesar das inúmeras pesquisas e Questionamentos Quanto à real efetividade do uso de medicamentos para tratar SCPD, em um ponto, todos os estudos concordam; o tratamento farmacológico dos sintomas neuropsiQuiátricos não-cognitivos deve ser indicado somente Quando medidas não-farmacológicas são ineficazes para o controle dos sintomas $^{(4,8,17)}$.

Este estudo não pretende abordar as formas de terapia farmacológica no manejo dos SCPD. Mas, é necessário ressaltar Que o uso de antipsicóticos (típicos e atípicos), ansiolíticos, anticonvulsivantes, antidepressivos e anticolinesterásicos têm sido estudados de forma maciça, nos diferentes tipos de demência, mostrando-se efetivos, particularmente, nos casos de distúrbios do humor, do sono e dos sintomas psicóticos.

O tratamento não-farmacológico dos SCPD compreende a abordagem ao paciente, família e/ou cuidadores. Existem vários tipos de tratamento Que serão de escolha conforma o estágio da doença, motivação do idoso e de seus cuidadores, recursos ambientais e financeiros disponíveis.

Uma forma de terapia não-farmacológica é a reabilitação cognitiva Que pode incluir treinos de orientação para a realidade, treinamento de memória, reminiscência e outros. Além da reabilitação cognitiva estão outras técnicas como arteterapia, terapias ocupacionais, dança, musicoterapia, exercícios, etc ${ }^{(8)}$.

Autores sugerem adaptações ambientais, instituição de rotinas e orientações ao cuidadores como o uso de música suave durante o banho, evitar, ao máximo, confrontos com o paciente e um rigoroso acompanhamento da data, hora e situação em Que os SCPD apareceram $^{(18.19)}$.

A Academia Brasileira de Neurologia recomenda programas educacionais e treinamento do cuidador somados ao suporte psicológico e dos serviços de saúde e, afirma, Que este tipo de intervenção melhora os níveis de estresse, retardam a institucionalização, e evitam, muitas vezes, o uso de fármacos ${ }^{(20)}$.

A equipe multidisciplinar é fundamental neste cuidado. $\mathrm{O}$ acompanhamento nutricional, fisioterápico e fonoaudiológico são necessários em diversos estágios da doença. E as intervenções de enfermagem são extremamente importantes em Questões Que giram em torno de hábitos de vida, uso correto dos medicamentos, cuidados com a pele, hidratação e no manejo das intercorrências clínicas. $\mathrm{O}$ atendimento familiar proporciona discussões, orientações e aconselhamentos Que visam à melhora do cuidado e a prevenção de problemas. Através desse atendimento é possível identificar de forma mais eficaz as necessidades do idoso demenciado e de seus cuidadores e então planejar estratégias de sucesso junto ao paciente.

\section{Atuação da Enfermagem Gerontológica junto ao Idoso Demenciado e seus Cuidadores no Manejo dos SCPD}

A enfermagem gerontológica brasileira se constitui num corpo de conhecimentos específicos, organizado recentemente, com indicações de suas primeiras publicações a partir da década de 70 . Não obstante, tem se ampliado em pesQuisa e participações ativas nas produções atuais acadêmicas e políticas. Hoje, a enfermagem gerontológica é definida pela Organização Pan-Americana de Saúde como um serviço de saúde Que incorpora aos conhecimentos específicos de enfermagem aQueles especializados sobre o processo 
de envelhecimento, para estabelecer no idoso e ao seu redor as condições Que permitam, entre outras, aumentar as condutas saudáveis e minimizar e compensar as perdas de saúde e as limitações relacionadas com o idoso.

Desta forma, está o profissional enfermeiro habilitado a integrar a equipe multidisciplinar, pautado na educação em saúde, contribuindo para o planejamento, realização e suporte para o cuidado e atendimento às necessidades desses pacientes, e em especial, nos pacientes demenciados, a promoção do funcionamento cognitivo e do bem-estar funcional ${ }^{(21)}$.

O impacto do diagnóstico de demência na família é extremamente perturbador. Poucas pessoas estão preparadas para lidar com a responsabilidade e sobrecarga Que é cuidar de um idoso demenciado, pois, em geral, existe um desconhecimento sobre a doença, como agir, como entender a pessoa afetada e seus próprios sentimentos.

Ao procurar atendimento para um idoso demenciado, grande parte dos familiares o fazem em virtude do paciente apresentar declínio importante na execução das atividades de vida diária ou porQue estão surgindo alterações do humor e comportamento, como agitação e agressividade ${ }^{(22)}$.

Apesar de alguns escassos estudos evidenciarem pouca efetividade nas intervenções de suporte e informações aos cuidadores $^{(23)}$, sabe-se que a informação adequada sobre o Que demência, como tratá-la e como manejar o paciente ajudará que este impacto inicial seja melhor compreendido, proporcionando a melhora da Qualidade de vida tanto do paciente Quanto de seus cuidadores. Sabemos do sofrimento pelo Qual passam essas famílias, principalmente nos anos subseQüentes ao diagnóstico, a adaptação às novas tarefas impostas pela doença e é nosso dever informar $\mathrm{e}$ buscar soluções Que, apesar de não serem definitivas, têm, comprovadamente minimizado, em muito, o sofrimento de pacientes e familiares ${ }^{(22)}$.

Um idoso demenciado e dependente implica em eleição de um cuidador principal, mudança nos papéis familiares e necessidade de um grupo de suporte. O idoso e sua família necessitam de uma rede de apoio ampla Que inclui desde o acompanhamento ambulatorial da pessoa doente até o suporte estratégico para Quem cuida ${ }^{(24)}$.

Em geral, o atendimento desse idoso ocorre em ambulatórios, por uma equipe multiprofissional composta por médicos, assistentes sociais e enfermeiros. Na equipe multiprofissional a enfermeira através do exame físico, coleta de dados e aplicação de instrumentos de avaliação cognitiva e funcional constrói um plano de cuidados intra e extradomiciliar, para cada idoso, individualmente. A enfermeira através da consulta de enfermagem deve identificar Quem é o cuidador principal, como está estruturada e como funciona a dinâmica familiar e se ela conta ou não com estruturas de suporte (social e econômica). Após essa avaliação diagnóstica a família deve ser convocada para esclarecimentos Quanto à patologia, tratamento e prognóstico. É importante Que toda a família se envolva neste momento, pois é Quando a enfermeira dá as primeiras orientações e sugere a redefinição de Questões familiares.

A partir da consulta inicial, consultas de retorno devem ser agendadas com o propósito não somente de avaliar o idoso e a evolução do processo demencial, mas também para identificar as dificuldades encontradas pela família e junto a ela buscar soluções e alternativas para extinguir, ou pelo menos, minimizar os problemas surgidos.

$\mathrm{Na}$ literatura geriátrica e gerontológica, bem como nos manuais desenvolvidos para cuidadores, os cuidados de enfermagem ganham destaque com capítulos específicos. Essa assistência, em geral, abrange cuidados com a pele, prevenção de úlceras de pressão, higiene corporal e oral, vestimenta, nutrição e hidratação e administração de medicamentos. Além destes cuidados percebo Que cabe a enfermeira orientar Quanto a estratégias de manejo dos sintomas comportamentais e psicológicos no idoso demenciado. Dentre estes comportamentos, os mais freqüentes são: furtar, esconder objetos; agitação; reações catastróficas; fugas; perambulações; ataQues de pânico; ausência de autocuidado; alucinações; delírios; autoviolência e depressão ${ }^{(12,21,23,24)}$. Para cada um desses problemas existem estratégias específicas Que devem ser construídas junto ao cuidador.

Essa orientação não é feita exclusivamente no ambiente ambulatorial. A enfermeira dentro de suas atribuições e competências na atenção ao idoso está apta a realizar grupos de auto-ajuda e suporte tanto para os idosos Quanto para seus cuidadores, além da possibilidade de fazer visitas domiciliares e encaminhamentos para outros profissionais que compõe a equipe gerontológica, como terapeutas ocupacionais e neuropsicólogos.

Planejar, executar, monitorar e avaliar planos de cuidados com idosos demenciados requer criatividade e paciência. A enfermeira deve encorajar a família a envolver-se ao máximo, entender e seguilo com dedicação. Desta forma, falhas são melhor identificadas, promovendo a revisão e modificação constante do plano de cuidados, uma vez Que com a evolução da doença a dependência torna-se cada vez maior e as demandas mudam.

\section{CONSIDERAÇÕES FINAIS}

É evidente, através da literatura pesQuisada, a importância da assistência de enfermagem prestada ao idoso demenciado Que apresenta sintomas comportamentais e psicológicos, bem como aos seus cuidadores. Essa assistência vai muito além dos cuidados básicos Que dirigimos aos idosos sem comprometimento cognitivo e depende da participação ativa da família.

A busca por estratégias para minimizar e manejar a situação de cuidado pode agregar o conhecimento e a experiência de enfermagem como uma importante contribuição para a gerontologia e neuropsiquiatria geriátrica, no sentido de visualizar e operacionalizar novos modelos de cuidado na assistência à saúde dos idosos ${ }^{25}$, no momento em Que estes profissionais em sua prática de assistir os seres humanos holisticamente estão aptos a atuar na identificação de problemas e estabelecer intervenções necessárias. Pois então é a enfermagem definida como ciência e arte de assistir ao ser humano no atendimento de suas necessidades básicas, tornando-o independente, Quando possível, pelo ensino do autocuidado, bem como manter, promover e recuperar a saúde em colaboração com outros profissionais ${ }^{(25)}$. 


\section{REFERÊNCIAS}

1. Caldas CP. Cuidando de uma pessoa idosa Que vivencia um processo de demência numa perspectiva existencial. In: Guerreiro T, Caldas CP. Memória e Demência: (re) conhecimento e cuidado. Rio de Janeiro: UERJ/UnATI; 200I. p. 129-206.

2. Veras RP. País jovem com cabelos brancos: a saúde do idoso no Brasil. Rio de Janeiro: Relume Dumará; 1994.

3. Papaléo Netto M. Tratado de gerontologia. São Paulo: Atheneu; 2007.

4. Tamai S. Tratamento dos transtornos do comportamento de pacientes com demência. Rev Bras PsiQuiatr 2002; 24 (supl I): 15-21.

5. Finkel SI, Costa e Silva I, Cohen G, Miller S, Sartorius N. Behavioural and psychological signs and symptoms of dementia: a consensus statement on current knowledge and implications for research and treatment. Int Psychogeriatr 1996; 8(suppl 3): 497-500.

6. Garrido R, Almeida OP. Distúrbios de comportamento em pacientes com demência - impacto sobre a vida do cuidador. Are Neuropsieuiatr 1999; 57(2-B): 427-34.

7. Caldas CP. Envelhecimento com dependência: responsabilidades e demandas da família. Cad Saúde Pública 2003; 19(3): 77381 .

8. Freitas EV. Tratado de geriatria e gerontologia. $2^{\text {a }}$ ed. Rio de Janeiro: Guanabara Koogan; 2006.

9. Jorm AF. The prevalence of dementia: a Quantitative integration of the literature. Acta Psychiatr Scan 1987; 76: 465.

10. Alzheimer's Association. Alzheimer's Disease facts and figures. [citado em 25 fev 2008]. Disponível em: http://www.alz.org/ national/documents/Report_2007FactsAndFigures.pdf

11. Veciana JMG. Tratamiento de los síntomas no cognitivos de la enfermedad de Alzheimer. Rev Neurol 2006; 42(8): 482-8.

12. Diana G. Aspetti metodologici e normativi dei trial clinici per i sintomi comportamentali e psicologici in corso di demenza. Ann Ist Sanità 2003; 39(2):267-74.

13. Lyketsos CG. Prevalence of neuropsychiatric symptoms in dementia and mild cognitive impairment: results from the cardiovascular helth study. JAMA 2002; 288(12): 1475-83.
14. Hwang TJ. Mild cognitive impairment is associated with characteristic neuropsychiatric symptoms. Alzheimer Dis Assoc Disord 2004; 8:17-21.

15. Geda YE, smith GE, Knopman DS. Genesis of neuropsychiatric symptoms in mild cognitive impairment. Int Psychogeriatr 2004; 16: $51-60$.

16. Bottino CMC. Prevalence of dementia and $\mathrm{MCI}$ in São Paulo, Brazil. Int Psychogeriatr 2005; 17:80.

17. Caramelli P, Bottino CMC. Tratando os sintomas comportamentais e psicológicos da demência (SCPD). I Bras Psieuiatr 2007; 56(2): 83-7.

18. Garrido R, Almeida OP. Distúrbios de comportamento em pacientes com demências - impacto sobre a vida do cuidador. Are Neuropsiquiatr 1999; 57(2-B): 427-34.

19. Ropacki AS, Jeste DV. Epidemiology of and risk factors for psychosis of Alzheimer's Disease: a review of 55 studies published from 1990 to 2003. Am I Psychiatry 2005; 162: 2022-30.

20. Engelhardt E. Tratamento da Doença de Alzheimer recomendações e sugestões do Departamento Científico de Neurologia Cognitiva e do Envelhecimento da Academia Brasileira de Neurologia. Are Neuropsieuiatr 2005; 63(4): 1104-12.

21. Figueiredo NMA, Tonini T, orgamizadores. Gerontologia: atuação da enfermagem no processo do envelhecimento. São Paulo: Yendis; 2006.

22. Caovilla VP, Canineu PR. Você não está sozinho. São Paulo: ABRAZ; 2002.

23. Thompson CA. Systematic review of information and support interventions for caregivers of people with dementia. BMC Geriatrics 2007; 7-18.

24. Caldas CP. Contribuindo para a construção da rede de cuidados: trabalhando com a família do idoso portador de síndrome demencial. Textos Envelhecimento 2002; 4(8): 39-56.

25. Gwyther LP. Care of Alzheimer's patients: a manual for nursing home staff. Whasington: American Health Care Associations and Alzheimer's Disease and Related Disorders Association; 1985. 